\title{
miR-320/ELF3 axis inhibits the progression of breast cancer via the PI3K/AKT pathway
}

\author{
ZHIQIANG ZHANG ${ }^{1 *}$, JINKU ZHANG $^{2 *}$, JINMEI LI $^{2}$, HUIJUAN GENG $^{3}$, \\ BINGJUAN ZHOU ${ }^{2}$, BINGXIN ZHANG ${ }^{2}$ and HONG $\mathrm{CHEN}^{2}$ \\ Departments of ${ }^{1}$ Thoracic Surgery and ${ }^{2}$ Pathology, Baoding First Central Hospital; \\ ${ }^{3}$ Department of Clinical Laboratory, Baoding Infectious Diseases Hospital, Baoding, Hebei 071000, P.R. China
}

Received June 27, 2019; Accepted February 5, 2020

DOI: $10.3892 / \mathrm{ol} .2020 .11440$

\begin{abstract}
There is increasing evidence demonstrating that disorders affecting microRNAs (miRs) influence tumorigenesis and progression, which results in a poor prognosis in patients with breast cancer (BC). In the present study, the precise molecular mechanism underlying the role of miR-320 in the progression of $\mathrm{BC}$ was investigated. Reverse transcription-quantitative PCR was conducted to determine mRNA expression, and western blot analysis was used to test protein levels. An MTT assay was conducted to detect cell viability and Transwell assays were used to analyze cell migration and invasion abilities. Furthermore, E74-like factor 3 (ELF3) protein density was tested via immunohistochemistry. Tumor volume was detected by xenograft tumor formation assay. The current results indicated that miR-320 expression was downregulated in $\mathrm{BC}$ tissues and cells, and was associated with a poor prognosis of patients with BC. Overexpression of miR-320 inhibited cell proliferation, migration and invasion via inhibition of the epithelial-mesenchymal transition and the PI3K/AKT signaling pathway in BC cells. Furthermore, it was revealed that the tumor size and weight were smaller in nude mice that had been transfected to overexpress miR-320. The luciferase reporter assay demonstrated the direct binding of miR-320 to the 3' untranslated region of ELF3 mRNA, which may further downregulate ELF3. Overall, the present results provided evidence that miR-320 may be a tumor suppressor in $\mathrm{BC}$, and that the miR-320/ELF3 axis regulated tumor progression via the PI3K/AKT signaling pathway, which may represent a novel treatment strategy for BC.
\end{abstract}

Correspondence to: Dr Jinku Zhang, Department of Pathology, Baoding First Central Hospital, 320 Changcheng North Street, Baoding, Hebei 071000, P.R. China

E-mail:ydncuia@163.com

*Contributed equally

Key words: microRNA-320, E74-like factor 3, breast cancer, PI3K/AKT

\section{Introduction}

Breast cancer (BC) is one of the most common types of cancer in women, and occurs in the breast gland epithelium $(1,2)$. In recent years, BC has accounted for $22.9 \%$ of worldwide cancer diagnoses in women, whilst China accounted for $12.2 \%$ of all new diagnoses of $\mathrm{BC}$ and $9.6 \%$ of global BC-associated mortalities $(3,4)$. Despite significant advances in drug sensitivity and surgical techniques, metastasis still occurs frequently and represents the leading cause of advanced BC-associated mortality (2). Recently, research investigating potential molecular markers associated with the early diagnosis of relapse and metastasis has gained traction. This may help to identify possible therapeutic targets for BC.

MicroRNAs (miRNAs/miRs) have been demonstrated to influence the progression of various cancer types and have consequently become a research focus. It has been revealed that miRNAs are associated with tumor development and target mRNAs, resulting in regulation of cell aggressiveness, migration ability, invasiveness and apoptosis $(5,6)$. Additionally, miR-320 has been demonstrated to be expressed aberrantly in various cancer types. For example, Lieb et al (7) revealed that miR-320 is associated with the diagnosis and clinical features of prostate cancer. miR-320 has also been reported to have an inhibitory effect on gastric cancer progression and serves as a novel biomarker for its diagnosis and prognosis (8). Furthermore, miR-320 suppresses BC cell proliferation and invasiveness (9); however, the precise mechanism underpinning the role of miR-320 in BC has not yet been determined.

E74 Like ETS Transcription Factor 3 (ELF3) is also called ESE-1, and is typically associated with epithelial carcinoma (10). It has been reported to serve important roles in tumor progression and embryonic development (11). Notably, in various cancer types, ELF3 expression is dysregulated. For example, it is upregulated in colorectal (12), prostate (13) and lung cancer (14), whereas it is downregulated in oral squamous cell carcinoma (15) and ovarian cancer (16). Of note, the PI3K/AKT signaling pathway is considered a canonical regulator of tumorigenesis, the phosphorylation levels of PI3K and AKT may reflect the change of PI3K/AKT signaling pathway (17). Previous studies have revealed that ELF3 is a negative regulator of epithelial-mesenchymal transition (EMT) 
in ovarian cancer cells (16). In addition, miR-320 expression has been revealed to be downregulated in $\mathrm{BC}$, but its precise molecular mechanism remains to be elucidated.

In the present study, the role of miR-320 and the exact mechanism underlying its role in modulating BC cell proliferation, invasiveness and migration ability were investigated. It was determined that miR-320 exerted an inhibitory effect on cell progression, and that ELF3 is a specific target of miR-320. Furthermore, it was revealed that miR-320 suppressed cell progression by regulating the PI3K/AKT signaling pathway. This indicated that the miR-320/PI3K/AKT signaling pathway may influence $\mathrm{BC}$ cell progression and may provide a novel therapeutic target for patients with $\mathrm{BC}$.

\section{Materials and methods}

Sample collection and cell culture. The present study was approved by the Ethics Committees of Baoding First Central Hospital (approval no. BD-2015-07-A0034). In total, 52 patients with breast cancer (all female) were selected as subjects for this study, which was conducted between January 2015 and December 2018 in Baoding First Central Hospital (Baoding, China). The patients' tumor tissue and adjacent normal tissues ( $\geq 5 \mathrm{~cm}$ from the tumor border) were removed surgically and collected. Samples were verified as BC by an experienced pathologist and fresh frozen in liquid $\mathrm{N}_{2}$ prior to use. The ages of the 52 BC patients ranged from 38-69 years, and 59 years was the median age. The entire study was compliant with the Helsinki Declaration.

RPMI-1640 medium (HyClone; GE Healthcare Life Sciences) containing 10\% FBS (Gibco; Thermo Fisher Scientific, Inc.), $100 \mu \mathrm{g} / \mathrm{ml}$ streptomycin and $100 \mathrm{U} / \mathrm{ml}$ penicillin (Beyotime Institute of Biotechnology) was used to maintain BC cell lines (MCF-7, SK-BR-3, MDA-MB-231 and Hs578T) and normal human mammary gland cells (Hs578Bst), which were purchased from American Type Culture Collection. The cells were incubated at $37^{\circ} \mathrm{C}$ with $5 \% \mathrm{CO}_{2}$ in a humidified chamber.

Cell transfection. miR-320 mimic, miR-320 inhibitor, control mimic and control inhibitor, ELF3 overexpressing pcDNA3.1-ELF3 plasmid (ELF3 vector) and their negative controls (control vector) were produced by Shanghai GenePharma Co., Ltd. The sequences were: miR-320 mimic, 5'-AAA AGCUGGGUUGAGAGGGCGA-3'; miR-320 inhibitors, 5'-UCGCCCUCUCAACCCAGCUUUU-3'; control mimic, 5'-UCACAACCUCCUAGAAAGAGUAGA-3'; and control inhibitor, 5'-UCUACUCUUUCUAGGAGG UUGUGA-3' (Shanghai GenePharma Co, Ltd). MCF-7 and SK-BR-3 cells $\left(2.0 \times 10^{5}\right)$ were inoculated into 6-well plates and transfected using Lipofectamine ${ }^{\circledR} 2000$ (Invitrogen; Thermo Fisher Scientific, Inc.) as per the recommended protocols (100 nM of miRNAs per sample). After transfection for $48 \mathrm{~h}$, the cells were collected for subsequent experiments. The reverse transcription-quantitative polymerase chain reaction (RT-qPCR) or western blotting assays were used to evaluate transfection efficiency.

$R T$-qPCR. TRIzol ${ }^{\circledR}$ reagent (Invitrogen; Thermo Fisher Scientific, Inc.) was used to extract total RNA from BC cells
(MCF-7 and SK-BR-3 cells) or tumor samples, following the manufacturer's instructions. The first-strand complementary DNA was synthesized using the ReverTraAce qPCR RT kit (Toyobo Life Science), according to the manufacturer's protocol. qPCR was performed using the 7500 FAST RT-PCR system (Thermo Fisher Scientific, Inc.). The $2^{-\Delta \Delta C q}$ (18) method was used to calculate and quantify the relative expression of miR-320 and ELF3 after standardization to the endogenous controls U6 and GAPDH, respectively. Reaction conditions are as follows: Pre-denaturation at $95^{\circ} \mathrm{C}$ for $10 \mathrm{~min}$, and 35 cycles of denaturation at $95^{\circ} \mathrm{C}$ for $10 \mathrm{sec}$, annealing at $60^{\circ} \mathrm{C}$ for $20 \mathrm{sec}$ and extending at $72^{\circ} \mathrm{C}$ for $2 \mathrm{~min}$. The sequences of primers were as follows: miR-320 forward, 5'-ACACTCCAG CTGGGAAAAGCTGGGTTGAG-3' and reverse, 5'-CTC AACTGGTGTCGTGGA-3'; ELF3 forward, 5'-CATGACCTA CGAGAAGCTGAGC-3' and reverse, 5'-GACTCTGGAGAA CCTCTTCCTC-3'; U6 forward, 5'-CTCGCTTCGGCAGCA CA-3' and reverse, 5'-AACGCTTCACGAATTTCGT-3'; and GAPDH forward, 5'-GCACCGTCAAGGCTGAGAAC-3' and reverse, 5'-ATGGTGGTGAAGACGCCAGT-3'.

Western blot analysis. RIPA lysis buffer with protease inhibitor (Beyotime Institute of Biotechnology) was used to extract total protein from MCF-7 and SK-BR-3 cells. Total protein concentration was calculated using the bicinchoninic acid (BCA) Protein assay kit (Pierce; Thermo Fisher Scientific, Inc.). A total of $50 \mu \mathrm{g} /$ lane of protein was loaded onto $8-15 \%$ polyacrylamide gels and then transferred to nitrocellulose membranes. Non-specific binding was blocked using 5\% non-fat milk for $2 \mathrm{~h}$ at room temperature. The primary antibodies were added to incubate the membrane at $4^{\circ} \mathrm{C}$ overnight, which was followed by incubation with horseradish-peroxidase (HRP)-conjugated secondary antibodies for $2 \mathrm{~h}$ at room temperature. Rabbit monoclonal ELF3 antibody (1:500; cat. no. ab133621), rabbit polyclonal E-cadherin antibody (1:500; cat. no. ab15148), rabbit monoclonal N-cadherin antibody (1:500; cat. no. ab92547), rabbit monoclonal vimentin antibody (1:500; cat. no. ab202030), rabbit monoclonal PI3K antibody (1:500; cat. no. ab32089), rabbit polyclonal phosphorylated (p)-PI3K antibody (1:500; cat. no. ab182651), rabbit polyclonal Akt antibody (1:500; cat. no. ab8805), rabbit monoclonal p-Akt antibody (1:500; cat. no. ab81283), rabbit polyclonal GAPDH antibody (1:500; cat. no. ab37168) and secondary goat anti-rabbit (HRP) IgG antibody (1:2,000; cat. no. ab6721) were all purchased from Abcam. An enhanced chemiluminescence kit (EMD Millipore) was used to detect the signals. The target protein:GAPDH expression level ratio was used to evaluate the relative target protein expression.

Transwell assay. Cell migration ability and invasiveness were measured using Transwell assays. In cell migration analysis, MCF-7 and SK-BR-3 cells $\left(1 \times 10^{6}\right)$ were seeded into the upper chamber ( $8-\mu \mathrm{m}$ pore size inserts). The RPMI-1640 medium in the upper chamber was serum-free, and the medium in the lower chamber contained $10 \%$ FBS which served as a chemoattractant. After incubation for $24 \mathrm{~h}$ at $37^{\circ} \mathrm{C}$, PBS was used to wash the inserts and a cotton swab was used to remove non-migrated cells. The migrated cells in the lower chamber were fixed using $100 \%$ methanol for $5 \mathrm{~min}$ at $4^{\circ} \mathrm{C}$, stained with $0.05 \%$ crystal violet for $10 \mathrm{~min}$ at $4^{\circ} \mathrm{C}$ and then imaged using a 
light microscope (magnification, x200; Olympus Corporation). The process of determining cell invasiveness was identical to cell migration analysis, except that the Transwell membranes were pre-coated with $1 \mathrm{mg} / \mathrm{ml}$ Matrigel at $4^{\circ} \mathrm{C}$.

MTT assay. The MTT assay (Sigma-Aldrich; Merck KGaA) was used to measure the viability of MCF-7 and SK-BR-3 cells. The cells were seeded into 96 -well plates at a density of $5 \times 10^{3}$ cells/well and then cultured in an incubator at $37^{\circ} \mathrm{C}$ with $5 \% \mathrm{CO}_{2}$. MTT $(20 \mu \mathrm{l})$ was added into each well to measure cell viability and incubated for another $4 \mathrm{~h}$ at $37^{\circ} \mathrm{C}$. After incubation, the medium was removed and $150 \mu 1 \mathrm{DMSO}$ was added into the wells to dissolve the formazan crystals at $37^{\circ} \mathrm{C}$. The cell viability was calculated by measuring the optical density at $490 \mathrm{~nm}$.

Xenograft experiment. A xenograft tumor formation assay was used for the tumor growth assay in vivo. A total of 16 female nude mice (3-5 weeks old, 18-22 g) were purchased from the Shanghai SLAC Laboratory Animal Co.,Ltd. All animal experiments were approved by the Ethics Committee of Baoding First Central Hospital. During the course of experiments, the mice were kept in pathogen-free animal facilities with controlled temperature and humidity, under a $12 \mathrm{~h}$ light/dark cycle, and with free access to food and water. The nude mice were randomly divided into two groups. MCF-7 cells $\left(1 \times 10^{6}\right)$ transfected with miR-320 mimic or miR-negative control were added into the RPMI-1640 medium (100 $\mu$ l, HyClone; GE Healthcare Life Sciences). Subsequently, 5x10 MCF-7 cells were injected into the right flank of nude mice subcutaneously. A vernier caliper was used to measure the xenograft tumor size every 4 days, and the following formula was applied to calculate the tumor volume: $0.5 \mathrm{x}$ tumor width $\mathrm{x}$ tumor length. At 28 days after inoculation, the mice were sacrificed and the tumors were used for further analysis.

Immunohistochemistry assay. Firstly, paraffin sections $(6-\mu \mathrm{m}$ thick) were fixed using $4 \%$ paraformaldehyde for $30 \mathrm{~min}$ at $37^{\circ} \mathrm{C}$ and then incubated with $3 \% \mathrm{H}_{2} \mathrm{O}_{2}$ in PBS for 15 min at $37^{\circ} \mathrm{C}$. Following blocking with $5 \%$ goat serum at temperature for $2 \mathrm{~h}$ at $37^{\circ} \mathrm{C}$, the primary antibody anti-ELF3 $(1: 1,000$, cat. no. ab133621, Abcam) was added and incubated for $48 \mathrm{~h}$ at $4^{\circ} \mathrm{C}$. Subsequently, the sections were incubated with biotinylated goat anti-rabbit IgG (1:500; cat. no. sc-2004; Santa Cruz Biotechnology, Inc.) for $1 \mathrm{~h}$ at $37^{\circ} \mathrm{C}$. The sections were stained using diaminobenzidine mixture (Beijing Solarbio Science \& Technology Co., Ltd.) for $30 \mathrm{~min}$ at $37^{\circ} \mathrm{C}$ and then dehydrated using a graded alcohol series, cleared using xylene and coverslipped using neutral balsam. Finally, the protein density per section was determined using Image Pros Plus 5.0 software (Media Cybernetics). Images were captured with a fluorescence microscope (IX71, Olympus Corporation).

Dual luciferase reporter assay. Bioinformatics tools (TargetScanHuman 7.1, http://www.targetscan.org/vert_71/) were used to predict the miR-320 binding sites of ELF3. The recombinant pMIR-reporter luciferase vector was applied for luciferase assays. The wild or mutant type of ELF3-3 untranslated region ( $3^{\prime} \mathrm{UTR}$; containing miR-320 binding sites) were synthesized and then cloned into the pMIR-reporter luciferase vector (Promega Corporation). MCF-7 cells were co-transfected with miR-320 mimic and vector using Lipofectamine $^{\circledR} 2000$ (Invitrogen; Thermo Fisher Scientific, Inc.). MCF-7 cells were seeded into 48-well plates at a density of $5 \times 10^{4}$ cells per well. After transfection for $48 \mathrm{~h}$ at $37^{\circ} \mathrm{C}$, the Dual Luciferase Reporter assay system (Promega Corporation) was used to measure the luciferase activity. The relative luciferase activity was normalized to Renilla luciferase activity $48 \mathrm{~h}$ after transfection.

Statistical analysis. Data are presented as the mean \pm SD and all experiments were repeated in triplicate. The difference between two groups was compared using the unpaired two-tailed Student's t-test, and one-way ANOVA followed by Bonferroni's post hoc analysis was used to measure the significance of comparisons among more than two groups. Pearson's correlation analysis was used to assess the correlation between miR-320 and ELF3 expression. The overall survival was calculated using Kaplan-Meier survival analysis and log-rank tests. SPSS v19.0 software (IBM Corp.) was used to perform statistical analyses. $\mathrm{P}<0.05$ was considered to indicate a statistically significant difference.

\section{Results}

miR-320 is downregulated in $B C$ and associated with a poor prognosis in patients with $B C$. Primarily, miR-320 expression in BC tissues was detected using RT-qPCR and the results revealed that miR-320 was significantly downregulated in BC tissues compared with in normal tissues (Fig. 1A). Subsequently, miR-320 expression in four BC cell lines was determined and it was demonstrated that miR-320 expression was downregulated significantly in all BC cell lines compared with in normal cells (Fig. 1B). Kaplan-Meier analysis revealed that the survival time of patients with high miR-320 expression was longer compared with patients in the low miR-320 expression group (Fig. 1C).

ELF3 is a target of miR-320 in BC cells. To investigate the mechanism underlying the role of miR-320 in the progression of BC, TargetScanHuman 7.1 was first applied to predict the potential targets of miR-320, and ELF3 was identified as one of the candidate targets (Fig. 2A). Subsequently, a dual-luciferase reporter assay was performed to further explore whether ELF3 represented a direct target of miR-320 in BC cells. It was revealed that the luciferase activity of ELF3-3'UTR-wild type was significantly decreased in MCF-7 and SK-BR-3 cells after transfection with a miR-320 mimic and pMIR-reporter luciferase vector, whereas there was no significant change in the mutant-type group (Fig. 2B), suggesting that ELF3 was a direct target of miR-320. Therefore, to confirm and further investigate the effects of miR-320 on ELF3 expression, a miR-320 mimic, miR-320 inhibitor, control mimic and control inhibitor were transfected to artificially regulate the expression levels of miR-320 in MCF-7 and SK-BR-3 cell lines. As shown in Fig. 2C, the introduction of miR-320 mimic could significantly upregulate the expression level of miR-320, while the miR-320 inhibitor could inhibit the expression of miR-320. Next, western blotting and RT-qPCR were carried out to investigate the effects of miR-320 on ELF3 expression. 

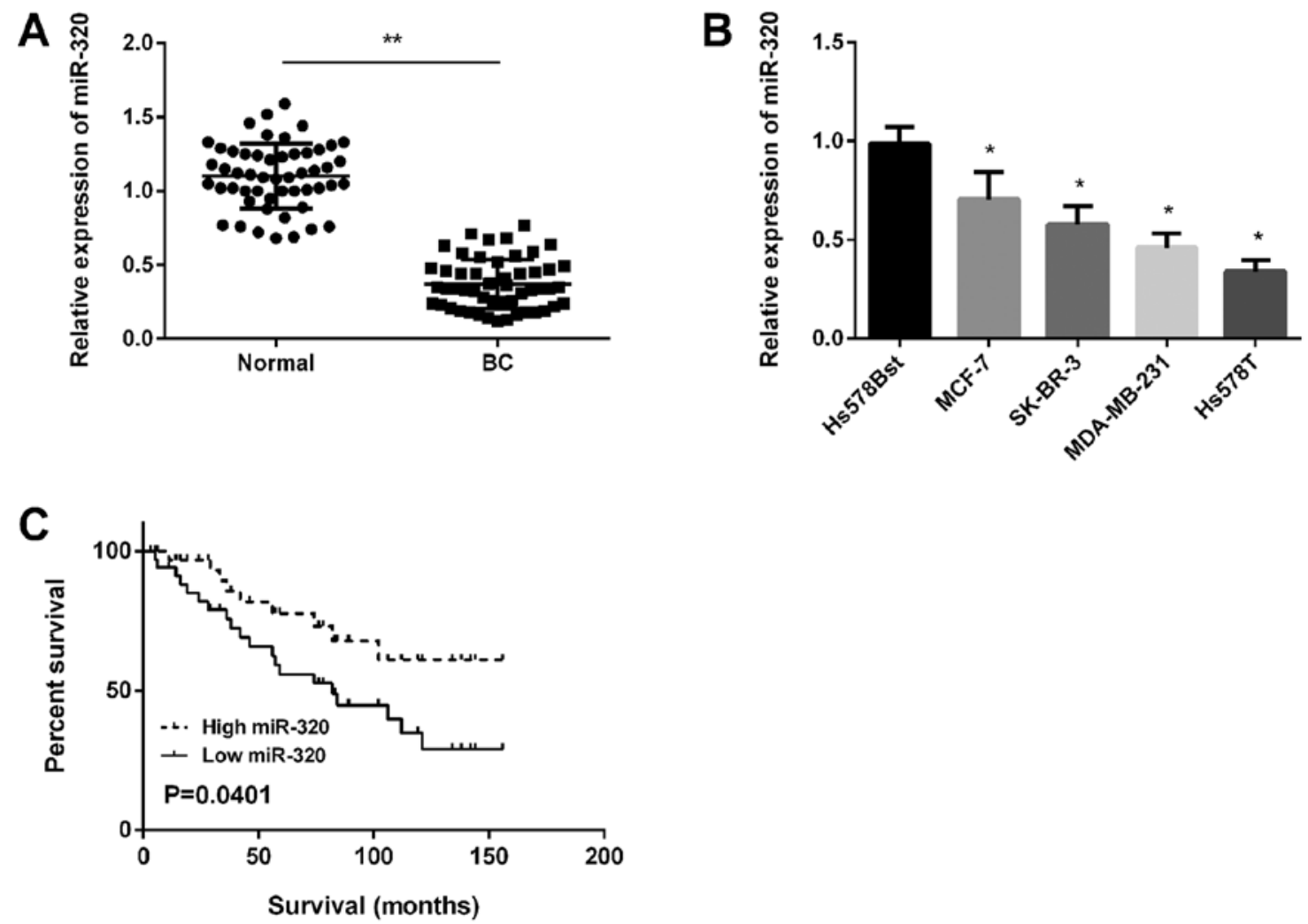

Figure 1. miR-320 expression in BC tissues. (A) miR-320 expression was downregulated in $\mathrm{BC}$ tissues compared with in normal tissues (n=52). ${ }^{* *} \mathrm{P}<0.01$. (B) miR-320 was downregulated in BC cells (MCF-7, SK-BR-3, MDA-MB-231 and Hs578T) compared with in normal human mammary gland Hs578Bst cells. "P<0.05 vs. Hs578T cells. (C) High expression of miR-320 was associated with longer overall survival time in patients with BC. BC, breast cancer; miR, microRNA.

It was demonstrated that ELF3 expression was suppressed by a miR-320 mimic, and elevated by miR-320 inhibitor in both BC cell lines (Fig. 2D and E). Pearson correlation analysis revealed a negative correlation between ELF3 and miR-320 expression in the 52 tumor samples (Fig. 2E; $R=-0.6769$; $\mathrm{P}<0.0001$ ), indicating that $\mathrm{miR}-320$ may regulate ELF3 expression negatively by binding to the 3'UTR of ELF3.

ELF3 is upregulated in BC tissues and associated with a poor prognosis in patients with $B C$. ELF3 expression was detected in BC tissues using immunohistochemistry and it was discovered that ELF3 protein was localized in the cytoplasm of BC tissues (Fig. 3A) and the staining intensity of ELF3 was higher in $\mathrm{BC}$ tissues compared with in normal tissues (Fig. 3B). Kaplan-Meier analysis revealed that the survival rate of patients with BC in the low-ELF3 expression group was markedly higher compared with that of patients in the high-ELF3 expression group (Fig. 3C).

ELF3 rescues the inhibitory effect of miR-320 on BC cell proliferation, migration ability and invasiveness. miR-320 mimic was successfully transfected into MCF-7 and SK-BR-3 cells to increase miR-320 expression levels, and the ELF3 vector was successfully transfected into MCF-7 and SK-BR-3 cells to increase the ELF3 expression level (Fig. 4A). MTT results determined that promoting miR-320 expression by transfection with a miR-320 mimic inhibited the proliferation of MCF-7 and SK-BR-3 cells, and that ELF3 overexpression partially reversed this effect (Fig. 4B and C). Transwell migration assays revealed that overexpression of miR-320 suppressed the migration ability of MCF-7 and SK-BR-3 cells, while ELF3 overexpression partially rescued this miR-320-mediated suppressive effect (Fig. 4D). The invasion assay revealed similar results; ELF3 expression was associated with promotion of the invasiveness of $\mathrm{BC}$ cells reduced by miR-320 (Fig. 4E). Taken together, the current results indicated that miR-320 inhibits BC cell proliferation, migration and invasiveness, and that ELF3 expression partially rescues the aforementioned inhibitory effects.

miR-320 inhibits tumor growth in nude mice. Subsequently, the effect of miR-320 expression on tumor growth was examined in vivo. MCF-7 cells transfected with a miR-320 mimic were injected into nude mice subcutaneously. The tumor volume was measured every 4 days. After 28 days, the mice were sacrificed, and the xenograft tumors were collected for further analysis. It was revealed that miR-320 overexpression inhibited the xenograft tumor growth (Fig. 5A) and the tumor cell xenografts exhibited a slower growth rate in the miR-320 mimic group compared with in the control group (Fig. 5B). In addition, the tumor weight in the miR-320 mimic group was significantly lower than in the control group (Fig. 5C).

miR-320/ELF3 axis modulates epithelial-mesenchymal transition (EMT) and PI3K/AKT signaling pathways in $B C$ cells. The influence of the miR-320/ELF3 axis on the EMT and PI3K/AKT signaling pathways was examined in BC cells. Western blotting was used to detect E-cadherin, N-cadherin, 
B

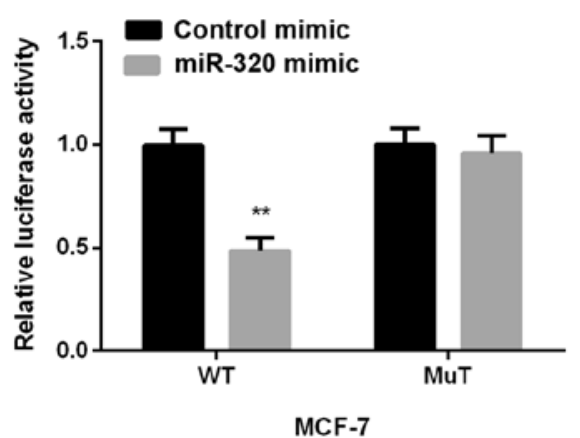

C

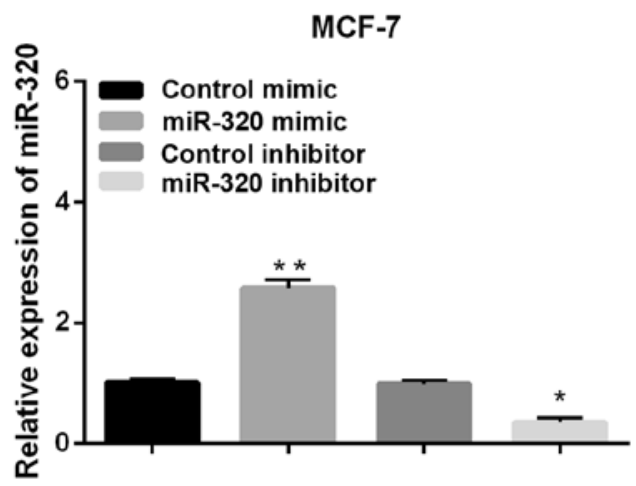

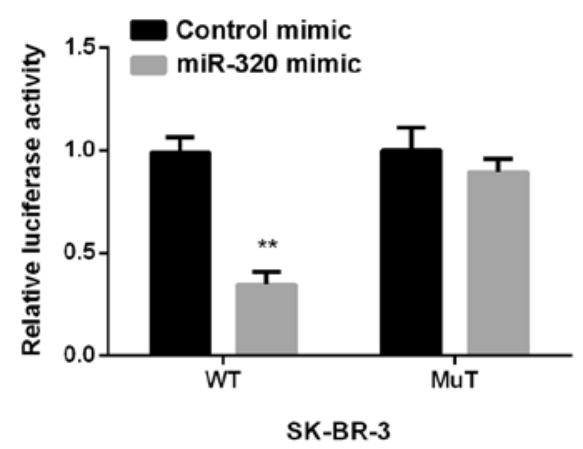

SK-BR-3

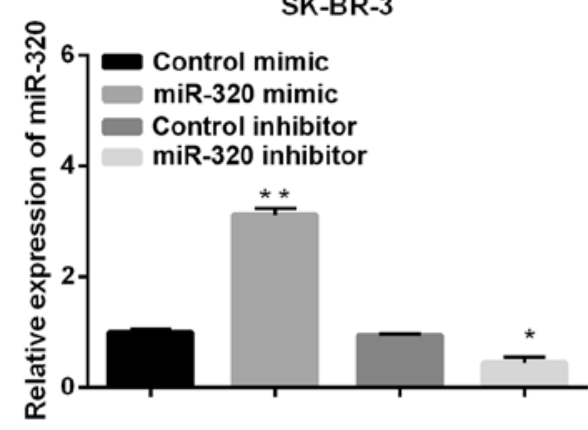

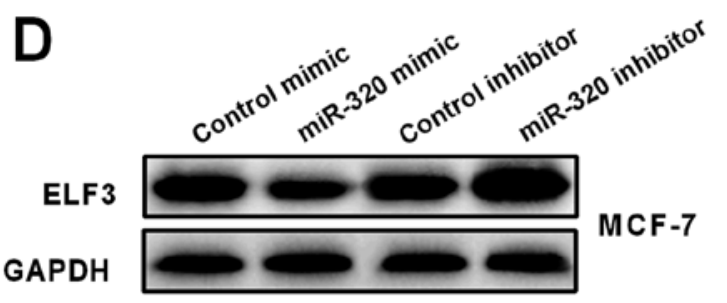

E

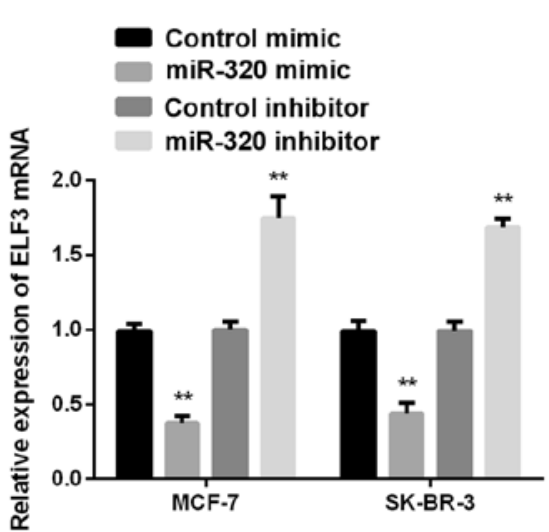

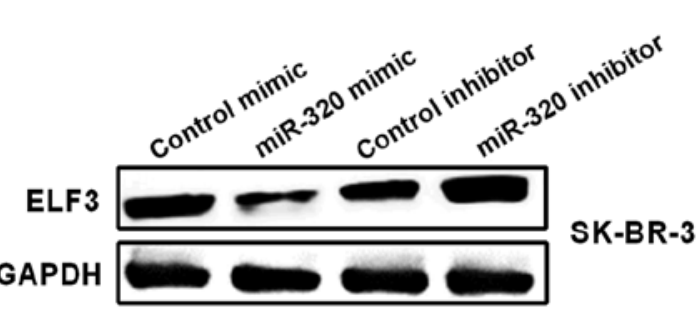

$\mathbf{F}$

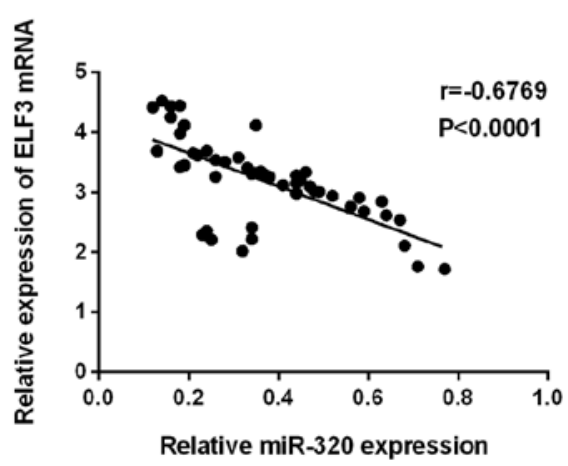

Figure 2. ELF3 is a target of miR-320 in BC cells. (A) Binding sites of miR-320 and ELF3 were predicted using TargetScanHuman 7.1. (B) Luciferase activities in MCF-7 and SK-BR-3 cells were downregulated after co-transfection with miR-320 mimic and ELF3-3'-UTR-wild type. (C) Expression levels of miR-320 in MCF-7 and SK-BR-3 cell lines were measured by reverse transcription-quantitative PCR following transfection with miR-320 mimic, miR-320 inhibitor, control mimic and control inhibitor. (D) ELF3 protein level and (E) ELF3 mRNA expression were downregulated or upregulated in MCF-7 and SK-BR-3 cells after treatment with miR-320 mimic or inhibitor. (F) ELF3 expression and miR-320 expression in $B C$ tissues $(n=52)$ were negatively correlated $(\mathrm{R}=-0.6769$; $\mathrm{P}<0.0001)$. " $\mathrm{P}<0.05,{ }^{* *} \mathrm{P}<0.01$ vs. normal tissues. $\mathrm{BC}$, breast cancer; miR, microRNA; ELF3, E74-like factor 3; 3'UTR, 3' untranslated region 

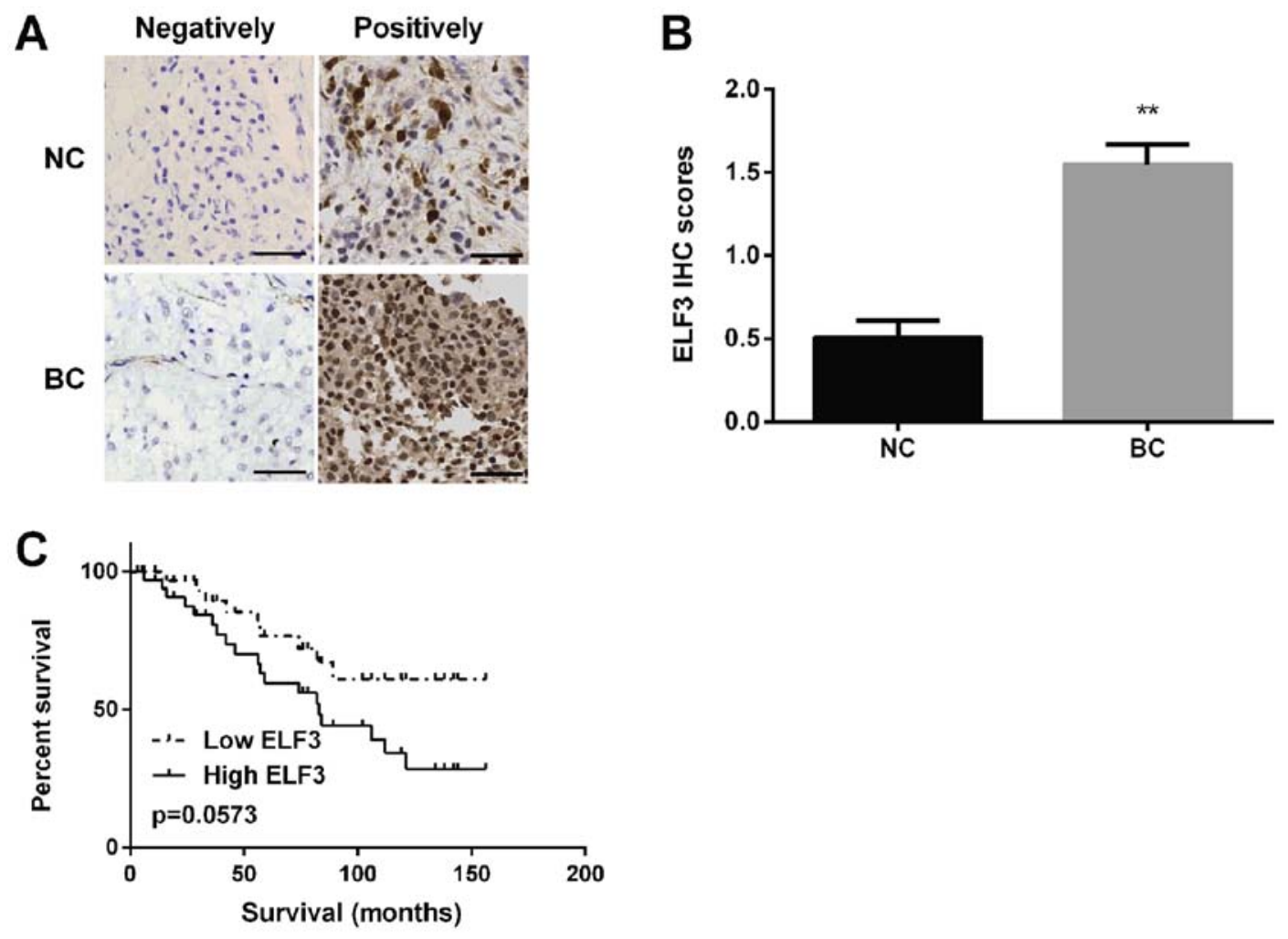

Figure 3. Expression of ELF3 in BC tissues. (A) Positive ELF3 protein expression was located in the cytoplasm of BC tissues. Scale bar, $100 \mu \mathrm{m}$. (B) ELF3 protein expression was increased in BC tissues compared with in normal tissues. (C) Low expression of ELF3 predicted a higher overall survival in patients with $\mathrm{BC} .{ }^{* *} \mathrm{P}<0.01$. BC, breast cancer; ELF3, E74-like factor 3; IHC, immunohistochemistry; NC, negative control.

vimentin, PI3K, p-PI3K, AKT and p-AKT protein levels in MCF-7 and SK-BR-3 cells, following transfection with miR-320 mimic or miR-320 mimic co-transfected with an ELF3 vector. As Fig. 6A indicates, the miR-320 mimic increased E-cadherin expression, while it decreased $\mathrm{N}$-cadherin and vimentin expression. Furthermore, ELF3 partially reversed the miR-320-mediated effect on EMT-associated markers. In addition, $\mathrm{p}-\mathrm{PI} 3 \mathrm{~K}$ and $\mathrm{p}-\mathrm{AKT}$ protein levels in the miR-320 mimic group were decreased compared with those in the control group, and ELF3 partially rescued the miR-320-mediated inhibitory effect on the PI3K/AKT signaling pathway (Fig. 6B), suggesting that miR-320 regulated the EMT and PI3K/AKT signaling pathway by targeting ELF3 in BC.

\section{Discussion}

miRNA dysregulation is closely associated with a variety of cancer types, and previous studies have revealed that miRNAs may be associated with tumor progression and prognosis (19-21). Comprehending the molecular mechanism underlying the influence of miRNAs in the progression of cancer may help identify novel treatment strategies. In the present study, it was discovered that miR-320 expression was downregulated in $\mathrm{BC}$ tissues and cells. Overexpression of miR-320 inhibited BC cell progression in vitro and tumor growth in vivo. Furthermore, ELF3 was identified as the target of miR-320, and miR-320/ELF3 was demonstrated to regulate the PI3K/AKT signaling pathway.

It has been demonstrated in large-scale studies that miRNAs influence BC progression by serving as oncogenes or tumor suppressors. For example, miR-590 has been revealed to suppress cell proliferation of $\mathrm{BC}$ via targeting of activating transcription factor 3 (22). Yan et al (23) reported that miR-125 suppresses BC progression by inhibiting BRCA1 associated protein 1 . Inversely, miR-19b serves as an oncogene in BC and enhances cell proliferation (24), and overexpression of miR-181a has been revealed to promote BC cell migration (25). Regarding studies of the role of miR-320 in BC, Luo et al (26) reported that increased miR-320 expression suppresses BC cell proliferation, invasiveness and migration via regulation of aquaporin 1 (AQP1). Furthermore, miR-320 inhibits BC cell progression via upregulation of SRY-box transcription factor 4 (9). These previous studies were consistent with the present study which reported that miR-320 expression was downregulated in BC tissues and cells, and miR-320 inhibited BC cell progression. Furthermore, to the best of our knowledge, it was reported for the first time that ELF3 represents a direct target of miR-320 in modulating BC progression.

Previously, it has been revealed that different miRNAs may regulate a common target gene or one miRNA may regulate multiple target genes in tumors (27). Numerous genes have been identified as targets of miR-320 in a wide variety of cancer types. For instance, AQP1 serves as a target of miR-320 to regulate BC progression (26). Forkhead box protein M1 influences the progression of glioma and is regulated by miR-320 (28). Notably, several studies have demonstrated that ELF3 is a target gene of certain miRNAs in other cancer types, and serves as an oncogene. Zhao et al (29) reported that ELF3 is upregulated in lung cancer and serves as the target of miR-320a in regulating lung 
A

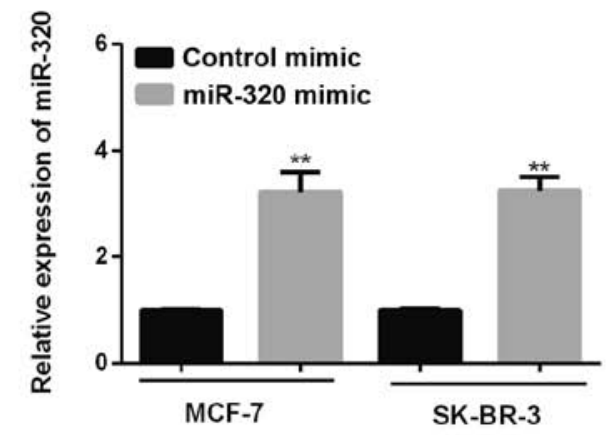

B
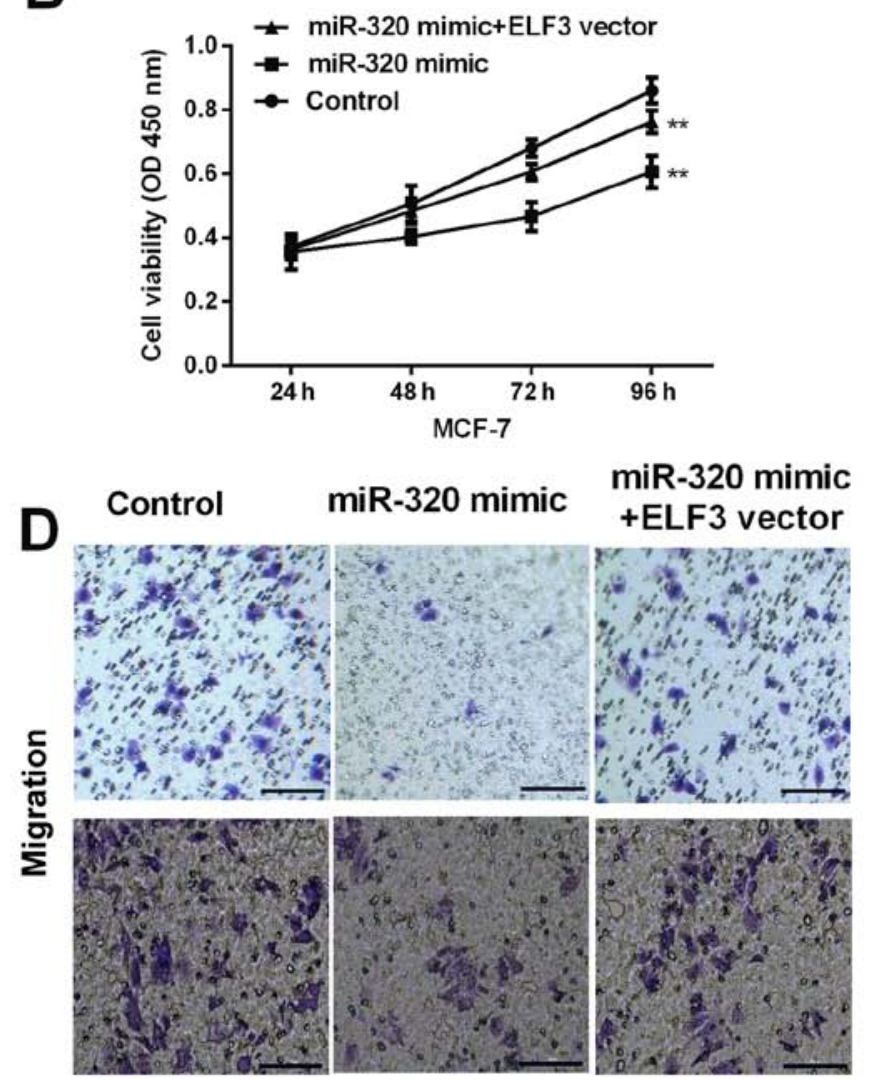

ฺุ
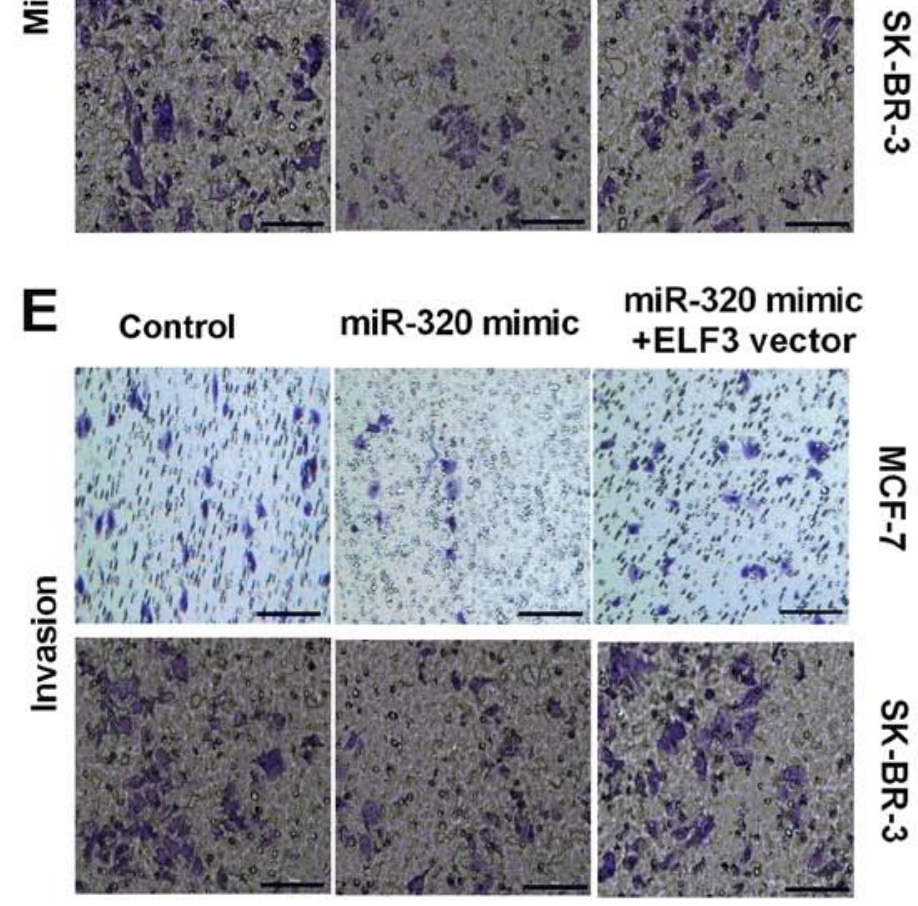

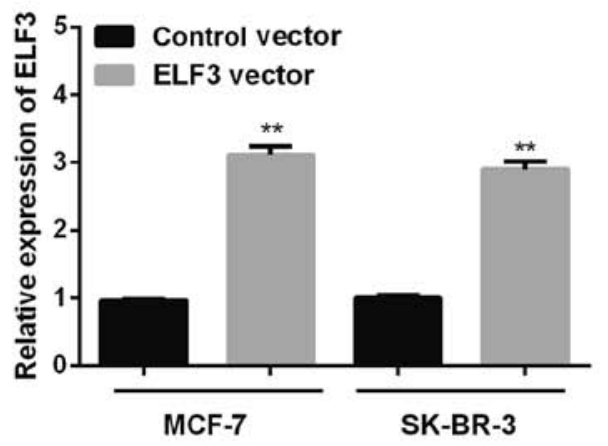

C
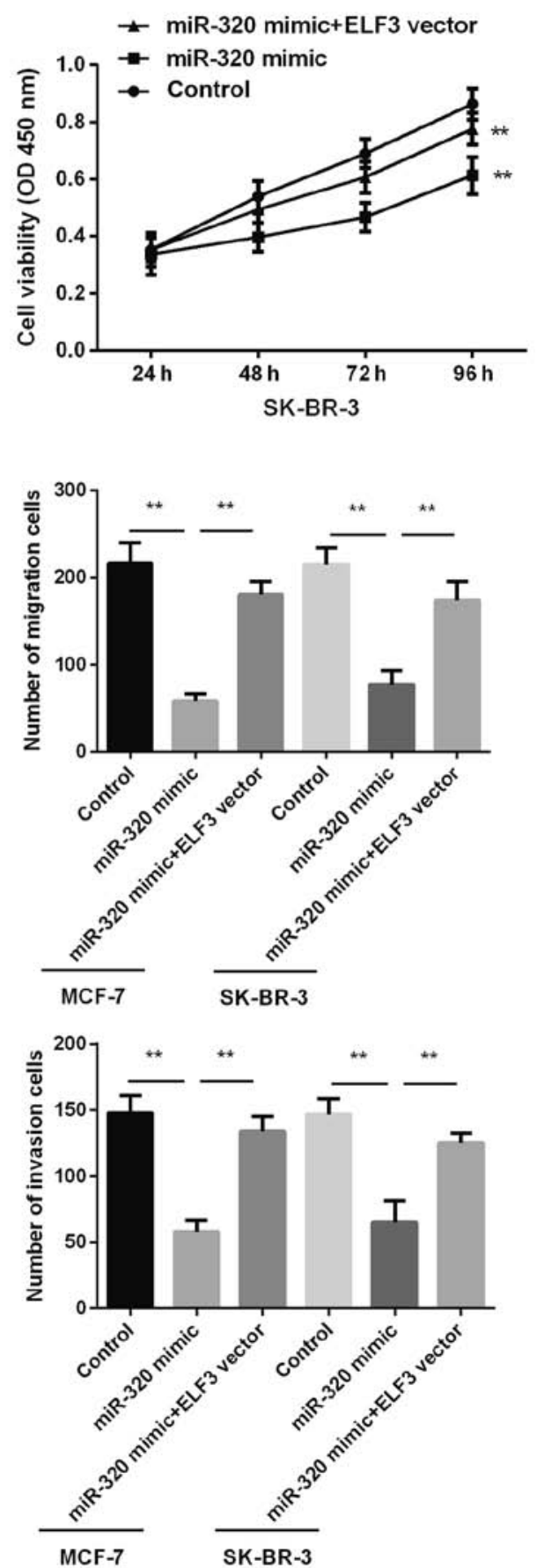

Figure 4. Inhibitory effect of miR-320 on BC cell proliferation, migration and invasion is reversed by ELF3. (A) miR-320 expression was higher in MCF-7 and SK-BR-3 cells after transfection with miR-320 mimic, and ELF3 expression was increased after transfection with ELF3 vector. (B) MCF-7 and (C) SK-BR-3 cell viability was decreased after transfection with miR-320 mimic, while it was increased when combined with ELF3 vector. (D) Cell migration and (E) invasion rates were decreased in MCF-7 and SK-BR-3 cells after transfection with miR-320 mimic, whereas they were increased after transfection with miR-320 mimic + ELF3 vector. Scale bars, $100 \mu \mathrm{m} .{ }^{* *} \mathrm{P}<0.01$. BC, breast cancer; miR, microRNA; ELF3, E74-like factor 3; OD, optical density. 


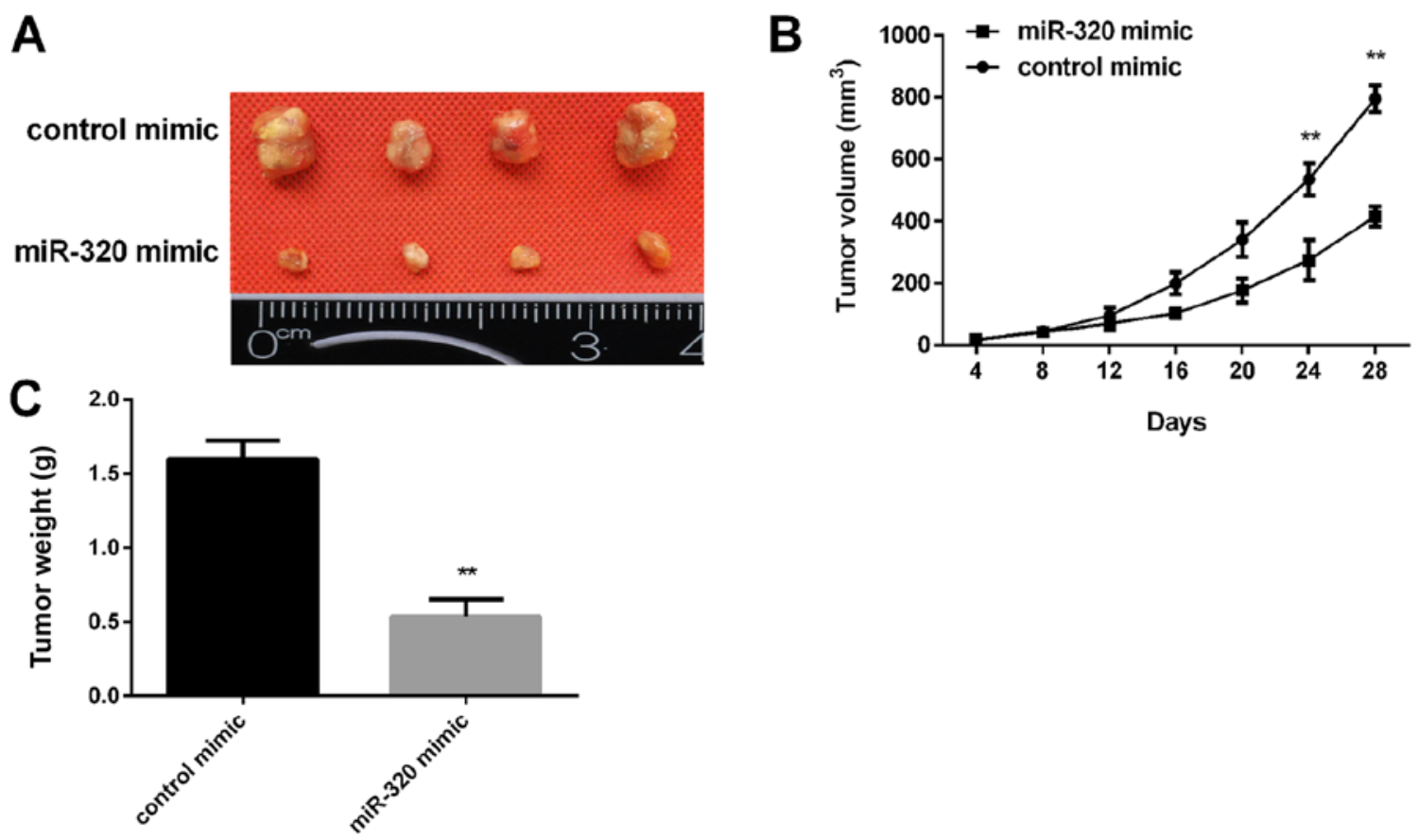

Figure 5. Effect of miR-320 on tumor growth in vivo. (A) Images of tumors overexpressing miR-320 mimic. (B) Growth rate of tumors transfected with miR-320 mimic was significantly lower compared with the control group at 24 and 28 days. (C) Tumor weight was significantly lower in the miR-320 mimic group compared with in the control group. ${ }^{* *} \mathrm{P}<0.01$ vs. control mimic group. miR, microRNA.
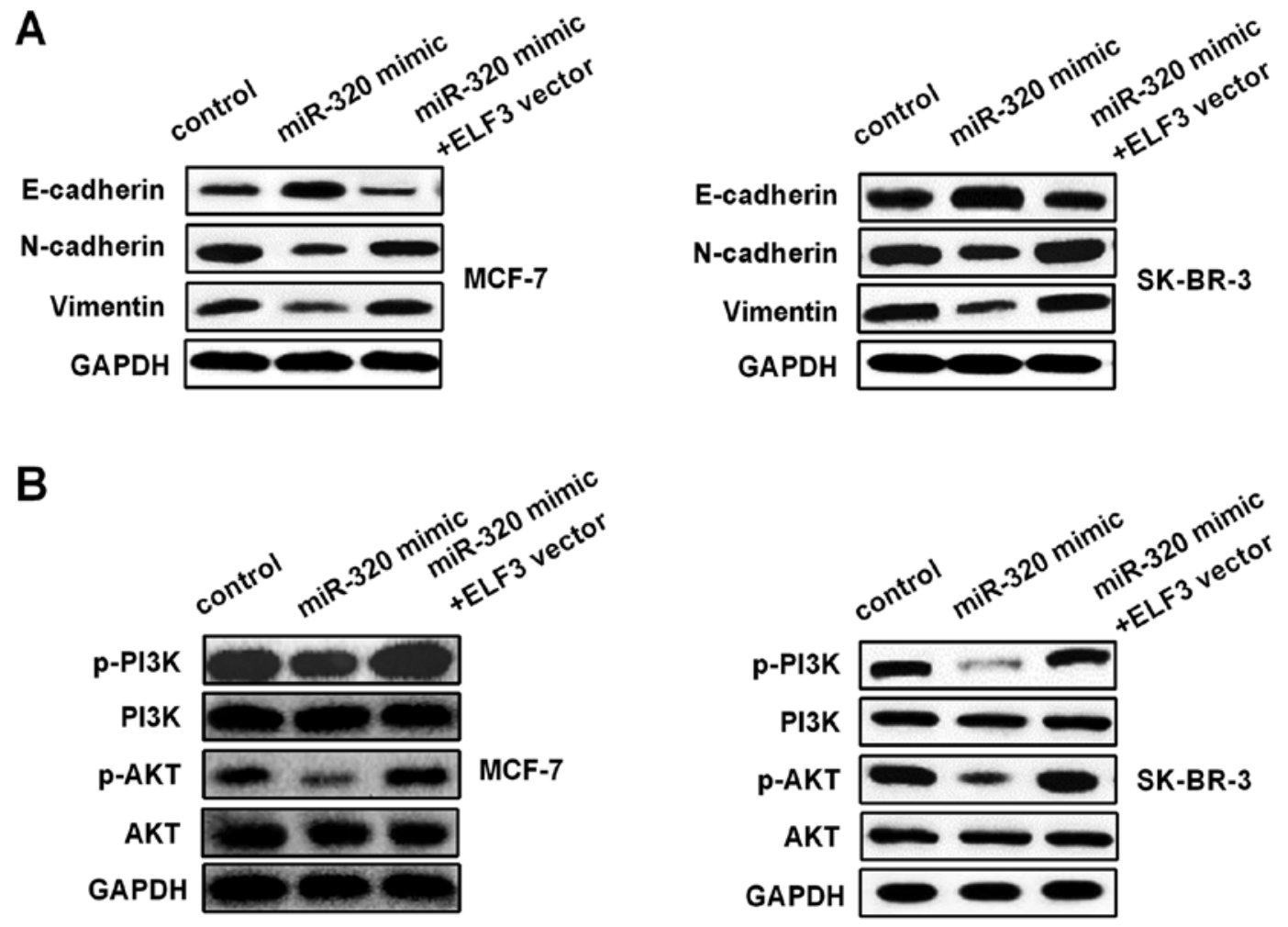

Figure 6. EMT and PI3K/AKT signaling pathway may be regulated by the miR-320/ELF3 axis in BC cells. (A) E-cadherin expression was upregulated, and $\mathrm{N}$-cadherin and vimentin expression were downregulated in MCF-7 and SK-BR-3 cells after transfection with miR-320 mimic. ELF3 partially reversed the effect on EMT-associated marker expression. (B) Phosphorylation of PI3K and AKT was downregulated in MCF-7 and SK-BR-3 cells after transfection with miR-320 mimic, and ELF3 expression partially reversed the effect on the PI3K/AKT pathway. EMT, epithelial-mesenchymal transition; miR, microRNA; ELF3, E74-like factor 3; p-, phosphorylated-.

cancer progression. Furthermore, ELF3 promotes EMT in hepatocellular carcinoma and has been identified as a target of miR-141 (30). In the present study, the results indicated that ELF3 was a target of miR-320 in BC. Upregulation of 
miR-320 inhibited ELF3 expression and there was a negative correlation between miR-320 and ELF3 expression. ELF3 was downregulated in $\mathrm{BC}$ and associated with the prognosis of patients with $\mathrm{BC}$.

The PI3K/AKT signaling pathway has been demonstrated to serve important roles in various pathophysiologic processes, including tumor growth, apoptosis, migration and invasiveness, as well as in EMT $(31,32)$. Certainly, the PI3K/AKT signaling pathway serves a central role in the progression of BC (33). It has previously been reported that the activation of ELF3 may inhibit the PI3K/AKT signaling pathway in lung cancer regulated by miR-320 $(14,29)$. The present study revealed that overexpression of miR-320 in MCF-7 and SK-BR-3 cells inhibited the PI3K/AKT signaling pathway, and ELF3 may reverse the inhibitory effect of miR-320 on the PI3K/AKT signaling pathway. The combined use of PI3K/AKT inhibitors may help to further elucidate the PI3K/AKT signaling pathway. The limitation of the present study was only exploring whether the PI3K/AKT signaling pathway influenced miR-320-regulated progression.

In conclusion, it was demonstrated that miR-320 targeted ELF3 to suppress BC cell progression via regulation of PI3K/AKT signaling. miR-320 may, therefore, represent a novel cellular therapeutic target for the treatment of patients with BC.

\section{Acknowledgements}

Not applicable.

\section{Funding}

The present study was supported by funding from the 2018 Hebei Provincial Key Research and Development Program Health Care and Biomedicine Special Project (grant no. $18277732 \mathrm{D})$.

\section{Availability of data and materials}

All data generated or analyzed during this study are included in this published article.

\section{Authors' contributions}

$\mathrm{ZZ}$ and $\mathrm{JZ}$ designed the study and performed the experiments. $\mathrm{ZZ}$, JL and HG analyzed and interpreted the data. JZ and BZho, BZha and HC analyzed the data. ZZ and JZ prepared the manuscript. All authors read and approved the final manuscript.

\section{Ethics approval and consent to participate}

The present study was approved by The Ethics Committees of Baoding First Central Hospital and all patients provided written informed consent.

\section{Patient consent for publication}

Patients or their legal guardians provided written informed consent for publication.

\section{Competing interests}

The authors declare that they have no competing interests.

\section{References}

1. Hong W and Dong E: The past, present and future of breast cancer research in China. Cancer Lett 351: 1-5, 2014.

2. Ren L, Li Y,Zhao Q, Fan L, Tan B, Zang A and Yang H: miR-519 regulates the proliferation of breast cancer cells via targeting human antigen R. Oncol Lett 19: 1567-1576, 2020.

3. DeSantis CE, Ma J, Goding Sauer A, Newman LA and Jemal A: Breast cancer statistics, 2017, racial disparity in mortality by state. CA Cancer J Clin 67: 439-448, 2017.

4. Zuo TT, Zheng RS, Zeng HM, Zhang SW and Chen WQ: Female breast cancer incidence and mortality in China, 2013. Thorac Cancer 8: 214-218, 2017.

5. Dinami R, Buemi V, Sestito R, Zappone A, Ciani Y, Mano M, Petti E, Sacconi A, Blandino G, Giacca M, et al: Epigenetic silencing of miR-296 and miR-512 ensures hTERT dependent apoptosis protection and telomere maintenance in basal-type breast cancer cells. Oncotarget 8: 95674-95691, 2017.

6. Zhao J and Jiang GQ: miR-4282 inhibits proliferation, invasion and metastasis of human breast cancer by targeting Myc. Eur Rev Med Pharmacol Sci 22: 8763-8771, 2018.

7. Lieb V, Weigelt K, Scheinost L, Fischer K, Greither T, Marcou M, Theil G, Klocker H, Holzhausen HJ, Lai X, et al: Serum levels of miR-320 family members are associated with clinical parameters and diagnosis in prostate cancer patients. Oncotarget 9: 10402-10416, 2017.

8. Zhang $\mathrm{H}$ and Lu W: LncRNA SNHG12 regulates gastric cancer progression by acting as a molecular sponge of miR-320. Mol Med Rep 17: 2743-2749, 2018.

9. Bai JW, Wang X, Zhang YF, Yao GD and Liu H: MicroRNA-320 inhibits cell proliferation and invasion in breast cancer cells by targeting SOX4. Oncol Lett 14: 7145-7152, 2017.

10. Oettgen P, Alani RM, Barcinski MA, Brown L, Akbarali Y, Boltax J, Kunsch C, Munger K and Libermann TA: Isolation and characterization of a novel epithelium-specific transcription factor, ESE-1, a member of the ets family. Mol Cell Biol 17: 4419-4433, 1997.

11. Neve RM, Parmar H, Amend C, Chen C, Rizzino A and Benz CC: Identification of an epithelial-specific enhancer regulating ESX expression. Gene 367: 118-125, 2006.

12. Wang JL, Chen ZF, Chen HM, Wang MY, Kong X, Wang YC, Sun TT, Hong J, Zou W, Xu J and Fang JY: Elf3 drives $\beta$-catenin transactivation and associates with poor prognosis in colorectal cancer. Cell Death Dis 5: e1263, 2014.

13. Longoni N, Sarti M, Albino D, Civenni G, Malek A, Ortelli E, Pinton S, Mello-Grand M, Ostano P, D'Ambrosio G, et al: ETS transcription factor ESE1/ELF3 orchestrates a positive feedback loop that constitutively activates NF- $\kappa \mathrm{B}$ and drives prostate cancer progression. Cancer Res 73: 4533-4547, 2013.

14. Wang H, Yu Z, Huo S, Chen Z, Ou Z, Mai J, Ding S and Zhang J: Overexpression of ELF3 facilitates cell growth and metastasis through PI3K/Akt and ERK signaling pathways in non-small cell lung cancer. Int J Biochem Cell Biol 94: 98-106, 2018.

15. Iwai S, Amekawa S, Yomogida K, Sumi T, Nakazawa M, Yura Y, Nishimune $Y$ and Nozaki M: ESE-1 inhibits the invasion of oral squamous cell carcinoma in conjunction with MMP-9 suppression. Oral Dis 14: 144-149, 2008.

16. Yeung TL, Leung CS, Wong KK, Gutierrez-Hartmann A, Kwong J, Gershenson DM and Mok SC: ELF3 is a negative regulator of epithelial-mesenchymal transition in ovarian cancer cells. Oncotarget 8: 16951-16963, 2017.

17. Ke K and Lou T: MicroRNA-10a suppresses breast cancer progression via PI3K/Akt/mTOR pathway. Oncol Lett 14: 5994-6000, 2017.

18. Livak KJ and Schmittgen TD: Analysis of relative gene expression data using real-time quantitative PCR and the 2(-Delta Delta C(T)) method. Methods 25: 402-408, 2001.

19. Ke SB, Qiu H, Chen JM, Shi W and Chen YS: MicroRNA-202-5p functions as a tumor suppressor in colorectal carcinoma by directly targeting SMARCC1. Gene 676: 329-335, 2018.

20. Quan H, Li B and Yang J: MicroRNA-504 functions as a tumor suppressor in hepatocellular carcinoma through inhibiting Frizzled-7-mediated-Wnt $/ \beta$-catenin signaling. Biomed Pharmacother 107: 754-762, 2018. 
21. Duan S, Dong X, Hai J, Jiang J, Wang W, Yang J, Zhang W and Chen C: MicroRNA-135a-3p is downregulated and serves as a tumour suppressor in ovarian cancer by targeting CCR2. Biomed Pharmacother 107: 712-720, 2018.

22. Rohini M, Gokulnath M, Miranda PJ and Selvamurugan N: miR-590-3p inhibits proliferation and promotes apoptosis by targeting activating transcription factor 3 in human breast cancer cells. Biochimie 154: 10-18, 2018.

23. Yan L, Yu MC, Gao GL, Liang HW, Zhou XY, Zhu ZT, Zhang CY, Wang YB and Chen X: miR-125a-5p functions as a tumour suppressor in breast cancer by downregulating BAP1. J Cell Biochem 119: 8773-8783, 2018.

24. Li C, Zhang J, Ma Z, Zhang F and Yu W: miR-19b serves as a prognostic biomarker of breast cancer and promotes tumor progression through PI3K/AKT signaling pathway. Onco Targets Ther 11: 4087-4095, 2018.

25. Taylor MA, Sossey-Alaoui K, Thompson CL, Danielpour D and Schiemann WP: TGF- $\beta$ upregulates miR-181a expression to promote breast cancer metastasis. J Clin Invest 123: 150-163, 2013.

26. Luo L, Yang R, Zhao S, Chen Y, Hong S, Wang K, Wang T, Cheng J, Zhang T and Chen D: Decreased miR-320 expression is associated with breast cancer progression, cell migration, and invasiveness via targeting Aquaporin 1. Acta Biochim Biophys Sin (Shanghai) 50: 473-480, 2018.

27. Palumbo T, Faucz FR, Azevedo M, Xekouki P, Iliopoulos D and Stratakis CA: Functional screen analysis reveals miR-26b and miR-128 as central regulators of pituitary somatomammotrophic tumor growth through activation of the PTEN-AKT pathway. Oncogene 32: 1651-1659, 2013
28. Li T, Ma J, Han X, Jia Y, Yuan H, Shui S and Guo D: MicroRNA-320 enhances radiosensitivity of glioma through down-regulation of sirtuin type 1 by directly targeting forkhead box protein M1. Transl Oncol 11: 205-212, 2018.

29. Zhao W, Sun Q, Yu Z, Mao S, Jin Y, Li J, Jiang Z, Zhang Y, Chen M, Chen $\mathrm{P}$, et al: miR-320a-3p/ELF3 axis regulates cell metastasis and invasion in non-small cell lung cancer via PI3K/Akt pathway. Gene 670: 31-37, 2018.

30. Zheng L, Xu M, Xu J, Wu K, Fang Q, Liang Y, Zhou S, Cen D Ji L, Han W and Cai X: ELF3 promotes epithelial-mesenchymal transition by protecting ZEB1 from miR-141-3p-mediated silencing in hepatocellular carcinoma. Cell Death Dis 9: 387, 2018.

31. Fang J, Ding M, Yang L, Liu LZ and Jiang BH: PI3K/PTEN/AKT signaling regulates prostate tumor angiogenesis. Cell Signal 19: 2487-2497, 2007.

32. Dong Y, Liang G, Yuan B, Yang C, Gao R and Zhou X: MALAT1 promotes the proliferation and metastasis of osteosarcoma cells by activating the PI3K/Akt pathway. Tumour Biol 36: 1477-1486, 2015.

33. Qiu N, He YF, Zhang SM, Zhan YT, Han GD, Jiang M, He WX, Zhou J, Liang HL, Ao X, et al: Cullin7 enhances resistance to trastuzumab therapy in Her2 positive breast cancer via degrading IRS-1 and downregulating IGFBP-3 to activate the PI3K/AKT pathway. Cancer Lett 464: 25-36, 2019.

(i) $\ominus$ This work is licensed under a Creative Commons Attribution-NonCommercial-NoDerivatives 4.0 International (CC BY-NC-ND 4.0) License. 